\title{
Algal and Cyanobacterial communities in two rivers of the province of San Luis (Argentina) subjected to anthropogenic influence
}

\author{
Comunidades de Algas e Cianobactérias em dois rios da província \\ de San Luis (Argentina) submetidos a influência antropogênica
}

Jorgelina Daruich, Susana Tripole, María Angélica Gil and Adriana Vallania

Área de Zoología, Departamento de Bioquímica y Ciencias Biológicas, Facultad de Química, Bioquímica y Farmacia, Universidad Nacional de San Luis - UNSL, Chacabuco 917, CP 5700, San Luis, Argentina e-mail: j.jorgelina@gmail.com; stripole@unsl.edu.ar; angil@unsl.edu.ar; vallania@unsl.edu.ar

\begin{abstract}
Aim: The use of biological indicators of pollution has increased in recent years as an alternative to the monitoring of water quality. Phytoplankton community selectively respond to different anthropogenic disturbances, such as water dams and the increase of nutrients coming from city centers, which leads to the eutrophication of the aquatic environment. The objective of this work was to evaluate the composition and the structure of the algal and Cyanobacterial communities in order to prove human influences by the presence of reservoirs with some degree of eutrophication and the impact of urbanization in two rivers at the Bebedero basin in San Luis province (Argentine). Methods: Four sites were sampled: two of them were placed before dams and villages $\left(\mathbf{V}_{1}\right)$ and $\left(\mathbf{P}_{\mathbf{1}}\right)$ and two after them $\left(\mathbf{V}_{\mathbf{2}}\right)$ and $\left(\mathbf{P}_{\mathbf{2}}\right)$. Each site was visited in every season of the year: summer, autumn, winter and spring. Qualitative and semi-quantitative phytoplankton samples were taken, and the frequency of occurrence was determined. Variations between pairs of sampling stations were analyzed through the Jaccard similarity and complementarity indices. Results: Ninety two taxa were identified, of which diatoms were the most frequent. The most affected station was $\mathrm{P}_{2}$ with high abundance, less diversity and equitability, whereas the species more tolerant to the presence of organic matter were Melosira varians, Navicula tripunctata, Oscillatoria limosa, Gomphonema parvulum and Coelastrum microporum, and some species of euglenophytas. Conclusion: Therefore, the structure and composition of the algal and Cyanobacterial communities allowed us to identify sections more sensitive to human-induced alterations.
\end{abstract}

Keywords: biological quality, algal communities, urbanization effect, regulated streams.

Resumo: Objetivo: $\mathrm{O}$ uso de indicadores biológicos de poluição tem aumentado nos últimos anos como alternativa de monitoramento da qualidade da água. A comunidade fitoplanctônica responde seletivamente a diferentes perturbaçóes antrópicas, como construção de barragens e aumento dos nutrientes em centros urbanos que levam à eutrofização de ambientes aquáticos. O objetivo deste trabalho foi avaliar a composição e a estrutura das comunidades de algas e cianobactérias, a fim de comprovar a influência humana através da presença de reservatórios com algum grau de eutrofização e do impacto da urbanização em dois rios na bacia do Bebedero da província de San Luis (Argentina). Métodos: Quatro estaçôes de coleta foram selecionados: duas antes dos reservatórios e aldeias (V1) e (P1) e duas após os mesmos (P2) e (V2). Cada estação foi visitada quatro vezes durante um ciclo anual: verão, outono, inverno e primavera. Foram coletadas amostras quantitativas e semiquantitativas do fitoplâncton e foi determinada a frequência de ocorrência. Foram analisados o grau de semelhança entre pares de estaçóes de coleta, utilizando o índice de similaridade de Jaccard e índice de complementaridade. Resultados: Foram identificados 92 táxons, sendo mais frequente as diatomáceas. P2 foi a estação mais afetada, mostrando alta abundância com menor diversidade e uniformidade. As espécies mais tolerantes à presença de matéria orgânica foram Melosira varians, Navicula tripunctata, Oscillatoria limosa, Gomphonema parvulum e Coelastrum microporum e algumas espécies de euglenophytas. Conclusáo: Portanto, a estrutura e composição das comunidades de algas e cianobactérias permitiu identificar secções mais sensíveis a alterações induzidas pelo homem.

Palavras-chave: qualidade biológica, comunidades de algas, efeito da urbanização, rio regulado. 


\section{Introduction}

The first descriptive studies of algal assemblages in different aquatic environments focused on the relationship among phytoplankton species as productivity indicators in lakes; for this reason, some indices and specific values defining productivity were established (Patrick and Reimer, 1975; Wetzel, 1981). Since phytoplankton constitute the first link in the trophic chain, their analysis of is of great interest in environmental. Additionally, their response to different environmental changes make them good water quality indicators, and they are a referent of the ecological status of the environment (Martínez de Fabricius, 2000; Pereira et al., 2000). The response of the phytoplankton community structure to different anthropogenic disturbances is a commonly used criterion to characterize water bodies (Mirande and Tracanna, 2005; Martínez de Fabricius et al., 2007). The growth of human population during the last decades, and the consequent expansion of towns and city centers, has generated solid and liquid wastes that affect water bodies, causing atrophic eutrophication (Dolbeth et al., 2003).

Changes in water quality are among the most frequent phenomena in eutrophic dams. These changes are mainly related to the high concentration of nutrients in water bodies, such as phosphorus and nitrogen (Ramírez García et al., 2007). The trophic state of a dam is directly related to the contributions they receive. Dams management is based on the stimulation of all those natural processes aimed at improving water quality while keeping the native population in balance with the environment, as well as placing a special emphasis on the combination of the physicochemical and biological factors. All of these factors determine the distribution of algal communities (Martínez de Fabricius et al., 2003, 2007).

Bacillariophyceae constitutes a group within algal communities with a rapid response to environmental changes (Rojo et al., 1994). They have are often used in studies aimed at monitoring the quality of water due to their ubiquitous distribution, high species diversity, and their siliceous frustules all enable the diatoms to function as sound environmental indicators (Reynolds, 1988, Cox, 1991).

Phytoplankton research in the province of San Luis has mainly focused on Bacillariophyceae (Maidana and Herbest, 1994; Daruich and Martínez de Fabricius, 2000, 2001, 2006;
Verteramo et al., 2001; Nievas et al., 2003; Daruich et al., 2003, 2005).

The objective of this work was to evaluate the composition and the structure of the algal and Cyanobacterial communities in order to prove human influences by the presence of reservoirs with some degree of eutrophication and the impact of urbanization in two rivers at the Bebedero basin in San Luis province (Argentine).

Our hypothesis is that the structure and composition of the algal community will allow us to identify sections more sensitive to human-induced alterations.

\section{Material and Methods}

\subsection{Study area}

The Bebedero basin is located at the centre of the province of San Luis. It is an endoreic basin, formed by two sub-basins which cover an area of $5500 \mathrm{~km}^{2}$, both of which are regulated and show different degrees of anthropogenic activity, since they run across tourist villages. The sub basin of $E l$ Volcan river, which is regulated by the Cruz the Piedra dam (built in 1941) supplied the water treatment plant of the city of San Luis until the decade of 1990 , when it started to be eutrophicated (Silva et al., 1995; Tognelli et al., 1997). The sub-basin of the Potrero river is regulated by the Potrero de los Funes dam, which was built in 1927, and today presents eutrophication signals (Almeida et al., 2007). These rivers drain over the eastern side of the San Luis mountains. This favors the development of a relatively important hydrographic network where the main constant flow rivers are born (Ceci and Cruz Coronado, 1981). The river systems of this area have annual variations in their watercourses (river flows), with droughts and flooding directly related to the rainfall rate. Rain occurs mainly in summer, from October to March, with an annual rainfall average between 500 and $650 \mathrm{~mm}$. This area corresponds to the Algarrobo district (Cabrera, 1976), and to the vegetal formation called Pastures and Mountain Forest (Anderson et al., 1970).

\subsection{Field and laboratory procedures}

Four sampling sites were chosen on the Bebedero basin. Two stations were placed before the location of the dam, and El Volcan and Potrero de los Funes villages: one was at El Volcan river $\left(\mathbf{V}_{\mathbf{1}}\right)$, and the other at Los Molles river $\left(\mathbf{P}_{\mathbf{1}}\right)$. The other two stations were placed after the dam: one at Las Chacras river $\left(\mathbf{P}_{2}\right)$, and another at the Cuchi Corral 
river $\left(\mathbf{V}_{2}\right)$ (Figure 1). Each station was visited four times along 2009 (summer, autumn, spring and winter) in order to check seasonal variations.

The hydraulic parameters of the river were recorded - width, current speed and flow. The physicochemical parameters were measured in situ with portable sensors: $\mathrm{pH}$ (accuracy $\pm 0.01 \mathrm{pH}$ ), conductivity (accuracy $\pm 1 \% \mu \mathrm{S} . \mathrm{cm}^{-1}$ ), temperature (accuracy $\pm 0.5^{\circ} \mathrm{C}$ ) (with CONSORT C532), and dissolved $\mathrm{O}_{2}\left(\mathrm{mg} . \mathrm{L}^{-1}\right)$ with CONSORT Z921.

Semi-quantitative and qualitative samples of phytoplankton were taken in each site. The collection of the samples was performed in the centre of the river. The taxonomic analyses were obtained using a mesh with an opening of 10 $\mu \mathrm{m}$ directly exposed into the water current for
20 minutes. The samples were fixed in situ with formaldehyde at $4 \%$. The semi-quantitative samples were stored in $500 \mathrm{ml}$ vessels using formaldehyde at $4 \%$ for their conservation. Quantification was made using a 40x magnification optic microscope, and a fixed number of randomly chosen fields were counted. Unicellular algae, colonies and filamentous algae were considered as individual units (Alveal et al., 1995).

For species identification, the following taxonomic references were used: Desikachary (1959), Prescott (1962), Patrick and Reimer (1966), Germain (1981), Krammer and Lange-Bertalot (1986, 1988, 1991a, b), Round and Bukhtiyarova (1996) and Stoermer et al., (1999). All the species described were ordered according to Bourrely (1981,

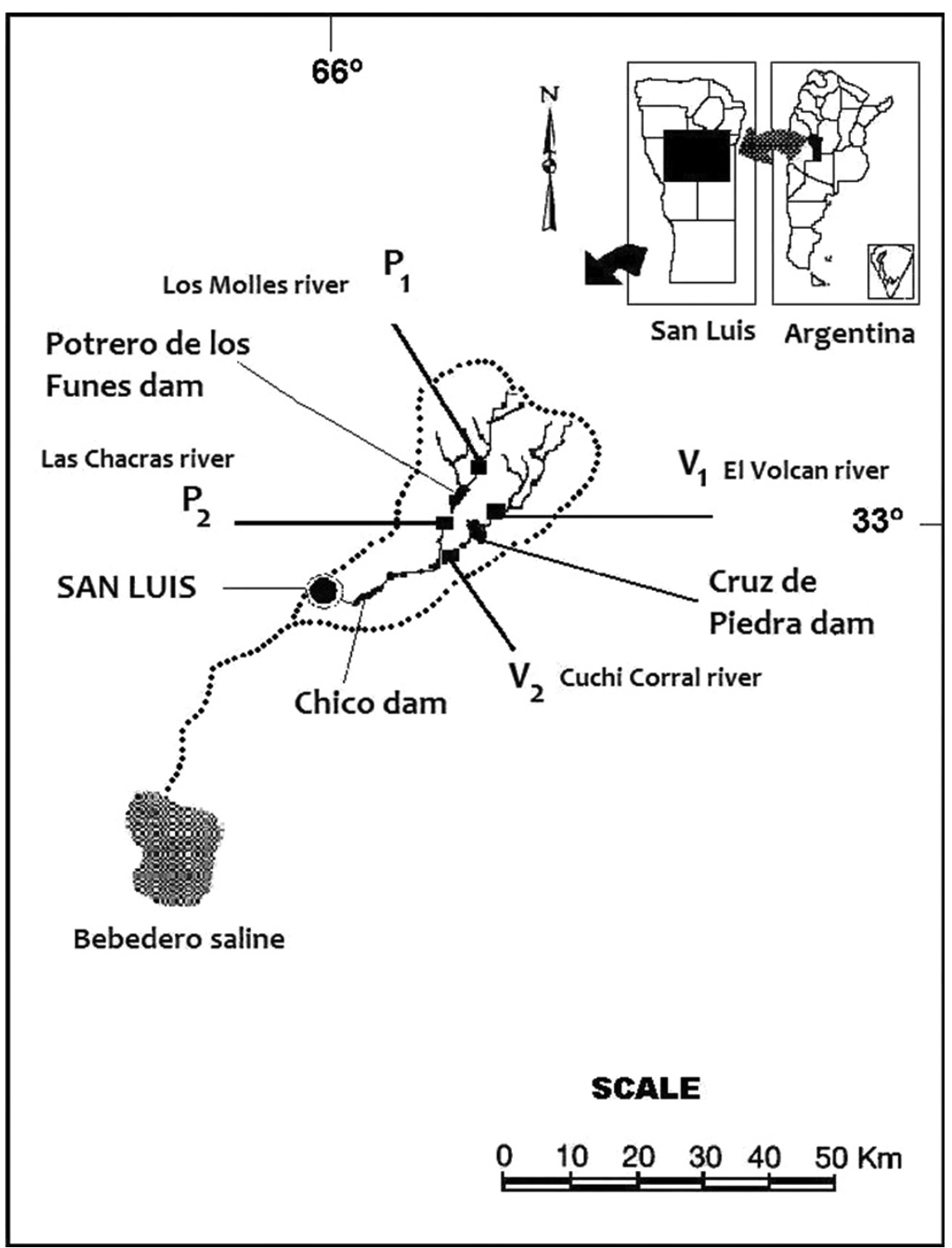

Figure 1. Location of the sampling sites in the Bebedero Basin: $\mathrm{V}_{1}$ El Volcán River, $\mathrm{V}_{2}$ Cuchi Corral River. $\mathrm{P}_{1}$ Los Mollles River and $\mathrm{P}_{2}$ Las Chacras River. 
1985, 1990) for Cyanophyceae, Chlorophyceae and Euglenophyceae, and according to Simmonsen (1979) for Bacillariophyceae.

Counts were performed through transects, using the method developed by Utermöhl (1958) and adapted by Villafañe and Reid (1995). Three transects were predetermined as horizontal, and edge effects were avoided. We used a slide and a coverslip of $24 \times 50 \mathrm{~mm}$. A $0.3 \mathrm{~mL}$ aliquot of mother sample was settled in the slides, which allowed for the observation of the material with a magnification of $400 \times$. We counted several preparations in order to obtain a constant average number of individuals (unicellular, colonies or filaments). For filamentous organisms, such as Oscillatoria, a unit was considered to be the equivalent to the length of a common single-celled organism, such as Synedra. Those species with a relative abundance of $\geq 1 \%$ were included in at least in one sample.

\subsection{Data analysis}

A table of presence-absence of species was made in order to determine the distribution of algae along the annual cycle. Following Paredes et al. (2007), taxa were considered as constant when they were recorded in more than $50 \%$ of the samples; they were determined to be common when they were found between $10 \%$ and $50 \%$ of the samples, and they were regarded as rare when they appeared in less than $10 \%$ of the samples. Density (org. $\mathrm{mL}^{-1}$ ), species richness (diversity $\alpha$ ), and ShannonWiener diversity index $\left(\mathrm{H}^{\prime}\right)$ were calculated in each sampling site. Jacquard's qualitative index of similarity (bits.ind ${ }^{-1}$.) was used to evaluate human influences through the degree of similarity in the composition between pairs of stations. This qualitative index considers the presence-absence of taxa by means of $\mathrm{Ij}=\mathrm{c} /(\mathrm{a}+\mathrm{b}+\mathrm{c}) \times 100$, where $\mathrm{c}$ is the number of species in both samples, $a$ is the number of species in sample 1 , and $b$ is the number of species in sample 2 . Values vary between 0 and 100 , the closer to 1 values are, the more species are lost and the stronger is the impact produced. $\beta$ ecological diversity was analyzed to differentiate the composition in each pair of sampling sites within each sub basin. To achieve this goal the complementarily index (IC) was used, which predicts when two communities are complementary (Colwell and Coddington, 1994; Moreno 2001). $\mathrm{B}=\mathrm{IC}_{\mathrm{AB}}=\mathbf{U} / \mathbf{S}, \mathbf{U}$ (number of unique species in any of the two sites) is calculated as: $\mathbf{a}+\mathbf{b}-\mathbf{2} \mathbf{c} . \mathbf{S}$ (the total richness for both sites combined ) whose formula is $\mathbf{S}=\mathbf{a}+\mathbf{b}-\mathbf{c}$., where $\mathbf{a}$ is the number of species in the area $A ; \mathbf{b}$ is the number of species in the area $\mathrm{B}$, and $\mathbf{c}$ is the number of common species in the both areas. Their values can vary between 0 and 1 .

Due to the fact that data do not have a normal distribution, the non-parametrical Kruskal-Wallis test was used with the physicochemical variables and the abundances of the collected samples in order to determine seasonal variations among the sampling sites. The Mann Whitney test was used to compare each pair of sampling sites.

\section{Results}

\subsection{Hydrological and physico-chemical parameters}

The average values of the hydrologic and physico-chemical variables of 4 sampling sites are presented in Table 1. Stations $\mathbf{V}_{2}$ and $\mathbf{P}_{2}$ registered

Table 1. Values (mean \pm standard deviation $[S D]$ ) of the measured physical, chemical and geographic variables in the study sites of the Bebedero basin (San Luis). V El Volcán River, V ${ }_{2}$ Cuchi Corral River, $\mathrm{P}_{1}$ Los Mollles River and $\mathrm{P}_{2}$ Las Chacras River.

\begin{tabular}{|c|c|c|c|c|}
\hline & $V_{1}$ & $V_{2}$ & $\mathbf{P}_{1}$ & $\mathbf{P}_{2}$ \\
\hline Elevation (m a. s. I.) & 992 & 799 & 1000 & 903 \\
\hline Stream Order & 2 & 3 & 2 & 3 \\
\hline Location & $\begin{array}{l}33^{\circ} 13^{\prime} 53.20^{\prime \prime} \mathrm{S} \\
66^{\circ} 12^{\prime} 17.43^{\prime \prime} \mathrm{W}\end{array}$ & $\begin{array}{l}33^{\circ} 173^{\prime} 40.64^{\prime \prime} \mathrm{S} \\
66^{\circ} 15^{\prime} 45.27^{\prime \prime} \mathrm{W}\end{array}$ & $\begin{array}{l}33^{\circ} 12^{\prime} 34.67^{\prime \prime} \mathrm{S} \\
66^{\circ} 13^{\prime} 46.722^{\prime \prime} \mathrm{W}\end{array}$ & $\begin{array}{l}33^{\circ} 14^{\prime} 32.15^{\prime \prime} \mathrm{S} \\
66^{\circ} 14^{\prime} 28.66^{\prime \prime} \mathrm{W}\end{array}$ \\
\hline $\begin{array}{l}\text { Discharge } \\
\left(\mathrm{m}^{3} \cdot \mathrm{seg}^{-1}\right)\end{array}$ & $\begin{array}{c}0.235 \pm 0.052 \\
0.18-0.30\end{array}$ & $\begin{array}{c}0.178 \pm 0.07 \\
0.08-0.25\end{array}$ & $\begin{array}{c}0.145 \pm 0.054 \\
0.1-0.22\end{array}$ & $\begin{array}{c}0.195 \pm 0.09 \\
0.06-0.28\end{array}$ \\
\hline $\begin{array}{l}\text { Velocity } \\
\left(\mathrm{m} . \text { seg }^{-1}\right)\end{array}$ & $\begin{array}{c}0.303 \pm 0.11 \\
0.22-0.46\end{array}$ & $\begin{array}{c}0.377 \pm 0.134 \\
0.26-0.57\end{array}$ & $\begin{array}{c}0.448 \pm 0.32 \\
0.22-0.9\end{array}$ & $\begin{array}{c}0.272 \pm 0.15 \\
0.15-0.48\end{array}$ \\
\hline $\mathrm{pH}$ & $\begin{array}{c}8.12 \pm 0.36 \\
7.6-8.4\end{array}$ & $\begin{array}{c}7.32 \pm 0.22 \\
7-7.5\end{array}$ & $\begin{array}{c}8.07 \pm 0.05 \\
8-8.1\end{array}$ & $\begin{array}{c}7.62 \pm 0.43 \\
7-8\end{array}$ \\
\hline $\begin{array}{l}\text { Conductivity } \\
\left(\mathrm{mS} . \mathrm{cm}^{-1}\right)\end{array}$ & $\begin{array}{c}447.3 \pm 332.1 \\
138-830\end{array}$ & $\begin{array}{c}972.5 \pm 219.9 \\
760-1200\end{array}$ & $\begin{array}{c}131.8 \pm 31.6 \\
110-177\end{array}$ & $\begin{array}{c}380.8 \pm 102.7 \\
310-533\end{array}$ \\
\hline $\begin{array}{l}\mathrm{O}_{2} \\
\left(\mathrm{mg} \cdot \mathrm{I}^{-1}\right)\end{array}$ & $\begin{array}{c}12.73 \pm 2.43 \\
9.7-14.7\end{array}$ & $\begin{array}{c}7.30 \pm 0.99 \\
6.05-8.2\end{array}$ & $\begin{array}{c}8.50 \pm 2.26 \\
6.4-11.7\end{array}$ & $\begin{array}{c}6.88 \pm 1.16 \\
5.72-7.97\end{array}$ \\
\hline $\begin{array}{l}\text { Water temperature } \\
\left({ }^{\circ} \mathrm{C}\right)\end{array}$ & $\begin{array}{c}17.4 \pm 2.23 \\
15-19.5\end{array}$ & $\begin{array}{c}17.3 \pm 2.12 \\
15-19.2\end{array}$ & $\begin{array}{c}16.3 \pm 4.93 \\
10.5-21.4\end{array}$ & $\begin{array}{c}15.18 \pm 4.67 \\
10.9-19.7\end{array}$ \\
\hline
\end{tabular}


the highest values of conductivity and the lowest values for dissolved oxygen. No significant differences were observed in relation to seasonal variations using the Kruskal Wallis.

Mann-Whitney test between pairs of stations showed no significant differences.

\subsection{Biological aspects}

The statistical comparison derived from the Kruskal-Wallis test of abundance at each sampling site showed no significant seasonal differences $(\mathrm{p}>0.05)$. These results were averaged for further calculations.

The phytoplankton community studied was composed of 92 species. According to its frequency, 33\% (Synedra ulna, Melosira varians and Gomphonema parvulum) were considered as constant; $65.2 \%$ were common species, and $31.3 \%$ were rare ones. No percentage variation within different seasons (Table 2) was observed. The less affected reference habitats presented the richest species of algae communities, with a tendency to a higher equitabilty (Table 3 ).

Table 2. Distribution and relative frequency of planktonic algae and Cyanobacteria in the sampling stations of the Bebedero basin. C: constant, c: commons and r: rare. $V_{1}$ El Volcán River, $\mathrm{V}_{2}$ Cuchi Corral River, $\mathrm{P}_{1}$ Los Mollles River and $\mathrm{P}_{2}$ Las Chacras River.

\begin{tabular}{|c|c|c|c|c|c|c|}
\hline \multicolumn{7}{|c|}{ Sampling sites Relative } \\
\hline Taxones & \multirow[t]{2}{*}{$\mathbf{v}_{1}$} & \multirow[t]{2}{*}{$\mathbf{V}_{2}$} & \multirow[t]{2}{*}{$\mathbf{P}_{1}$} & \multirow[t]{2}{*}{$\mathbf{P}_{2}$} & \multicolumn{2}{|c|}{ frequency $(\%)$} \\
\hline $\begin{array}{l}\text { Chroococcales } \\
\text { Chroococcaceae }\end{array}$ & & & & & & \\
\hline Chroococcus turgidus (Kütz.) Näg. & & $x$ & & $x$ & 12.5 & c \\
\hline Chroococcus $\mathrm{sp}_{1}$ & $\mathrm{x}$ & $\mathrm{X}$ & & $\mathrm{x}$ & 25 & c \\
\hline Chroococcus $\mathrm{sp}_{2}$ & & & $\mathrm{x}$ & $\mathrm{x}$ & 18.75 & c \\
\hline Gloeocapsa sp. & & & & $\mathrm{x}$ & 12.5 & c \\
\hline Merismopedia glauca (Ehren.) Näg. & & $\mathrm{x}$ & & & 6.25 & $\mathrm{r}$ \\
\hline $\begin{array}{c}\text { Hormogonales } \\
\text { Nostocaceae }\end{array}$ & & & & & & \\
\hline $\begin{array}{l}\text { Dolichospermum sp. } \\
\text { Oscillatoriaceae }\end{array}$ & & $x$ & & $x$ & 12.5 & c \\
\hline Artrospira sp. & & $x$ & & & 12.5 & c \\
\hline Lyngbya sp. & $\mathrm{x}$ & & & $\mathrm{x}$ & 12.5 & c \\
\hline Oscillatoria limosa Agardh & $\mathrm{x}$ & $\mathrm{x}$ & $\mathrm{x}$ & $\mathrm{x}$ & 68.75 & C \\
\hline Oscillatoria tenuis Agardh & $\mathrm{x}$ & $\mathrm{x}$ & $\mathrm{x}$ & $\mathrm{x}$ & 43.75 & c \\
\hline Oscillatoria sp & & & $\mathrm{x}$ & $\mathrm{x}$ & 18.75 & c \\
\hline $\begin{array}{l}\text { Oscillatoria } \mathrm{sp}_{1} \\
\text { CHLOROPHYCEAE } \\
\text { Volvocales } \\
\text { Chlamydomonadaceae }\end{array}$ & & & $x$ & $x$ & 18.75 & c \\
\hline Chlamydomonas sp. & & & & $x$ & 6.25 & $r$ \\
\hline $\begin{array}{l}\text { Chlorococcales } \\
\text { Oocystaceae }\end{array}$ & & & & & & \\
\hline Ankistrodermus sp. & & & & $x$ & 6.25 & $\mathbf{r}$ \\
\hline $\begin{array}{l}\text { Oocystis sp. } \\
\text { Scenedesmaceae }\end{array}$ & $\mathrm{x}$ & $\mathrm{x}$ & $\mathrm{x}$ & & 25 & c \\
\hline Coelastrum microporum Nägeli & & & & $x$ & 6.25 & $\mathbf{r}$ \\
\hline Crucigenia sp. & & $x$ & & $x$ & 18.75 & c \\
\hline Scenedesmus acutus Meyen & & $x$ & $x$ & & 18.75 & c \\
\hline Scenedesmus acutus f. costulatus (Chod.) Uherk. & & & $x$ & & 6.25 & $\mathbf{r}$ \\
\hline $\begin{array}{l}\text { Scenedesmus sp. } \\
\text { Hydrodyctaceae }\end{array}$ & & & $x$ & & 18.75 & c \\
\hline Monactinus simplex (Meyen) Corda, Alm. & & & & $x$ & 6.25 & $r$ \\
\hline Stauridium tetras (Ehren.) Hegew. & & & & $x$ & 12.5 & c \\
\hline $\begin{array}{l}\text { Ulothrichales } \\
\text { Ulothrichaceae }\end{array}$ & & & & & & \\
\hline Ulotrix sp. & $\mathrm{x}$ & $\mathrm{x}$ & $\mathrm{x}$ & & 31.25 & c \\
\hline
\end{tabular}


Table 2. Continued...

\begin{tabular}{|c|c|c|c|c|c|c|}
\hline \multicolumn{7}{|c|}{ Sampling sites Relative } \\
\hline Taxones & \multirow[t]{2}{*}{$\mathrm{V}_{1}$} & \multirow[t]{2}{*}{$\mathbf{V}_{2}$} & \multirow[t]{2}{*}{$\mathbf{P}_{1}$} & \multirow[t]{2}{*}{$\mathbf{P}_{2}$} & \multicolumn{2}{|c|}{ frequency $(\%)$} \\
\hline Oedogoniaceae & & & & & & \\
\hline Oedogonium sp. & $\mathrm{X}$ & & & & 6.25 & $\mathbf{r}$ \\
\hline $\begin{array}{l}\text { Siphonocladales } \\
\text { Cladophoraceae }\end{array}$ & & & & & & \\
\hline Cladophora glomerata (Linn.) Kütz. & $\mathrm{X}$ & $\mathrm{X}$ & & & 18.75 & c \\
\hline $\begin{array}{l}\text { Zygnematales } \\
\text { Zygnemataceae }\end{array}$ & & & & & & \\
\hline Mougeotia sp. & $\mathrm{X}$ & $x$ & & & 18.75 & c \\
\hline Spirogyra sp. & $\mathrm{X}$ & $\mathrm{X}$ & $\mathrm{X}$ & $x$ & 43.75 & c \\
\hline $\begin{array}{l}\text { Zygnema sp. } \\
\text { Desmidiaceae }\end{array}$ & $\mathrm{X}$ & & $\mathrm{X}$ & & 25 & c \\
\hline Closterium acutum Bréb. in Ralfs & & & $\mathrm{X}$ & & 6.25 & $\mathbf{r}$ \\
\hline Closterium moniliferum (Bory) Ehren.ex Ral. & $\mathrm{X}$ & $\mathrm{X}$ & $\mathrm{X}$ & & 37.50 & c \\
\hline Cosmarium botrytis Meneg. ex Ral. & $\mathrm{X}$ & $\mathrm{X}$ & $\mathrm{X}$ & & 25 & c \\
\hline Cosmarium $\mathrm{sp}_{1}$. & & & & $x$ & 6.25 & $\mathbf{r}$ \\
\hline $\begin{array}{l}\text { Staurastrums } \mathrm{sp}_{1} . \\
\text { EUGLENOPHYCEAE } \\
\text { Euglenales } \\
\text { Euglenaceae }\end{array}$ & & $\mathrm{X}$ & $\mathrm{X}$ & $x$ & 25 & c \\
\hline Euglena $\mathrm{sp}_{1}$. & & $x$ & & $x$ & 25 & c \\
\hline Euglena $\mathrm{sp}_{2}$. & & $\mathrm{X}$ & & & 12.5 & $\mathbf{r}$ \\
\hline Phacus sp. & & & $\mathrm{X}$ & $x$ & 18.75 & c \\
\hline $\begin{array}{l}\text { DINOPHYCEAE } \\
\text { Peridiniales } \\
\text { Ceratiaceae }\end{array}$ & & & & & & \\
\hline Ceratium hirundinella (O.F. Müll.) Bergh & $\mathrm{X}$ & & $\mathrm{X}$ & & 25 & c \\
\hline Peridiniacea & & & & & & \\
\hline Peridinium sp. & & $X$ & & & 6.25 & $\mathbf{r}$ \\
\hline $\begin{array}{l}\text { BACILLARIOPHYCEAE } \\
\text { Centrales } \\
\text { Coscinodiscinae } \\
\text { Thalssiosiraceae }\end{array}$ & & & & & & \\
\hline $\begin{array}{l}\text { Cyclotella meneghiniana Kütz. } \\
\text { Melosiraceae }\end{array}$ & $\mathrm{X}$ & $\mathrm{X}$ & & $\mathrm{X}$ & 25 & c \\
\hline Aulacoseira granulata (Ehren.) Sim. & $\mathrm{X}$ & $\mathrm{X}$ & & & 12.5 & c \\
\hline Melosira varians Ag. & $X$ & $\mathrm{X}$ & $X$ & $x$ & 62.50 & c \\
\hline $\begin{array}{l}\text { Pennales } \\
\text { Araphidinae } \\
\text { Diatomaceae }\end{array}$ & & & & & & \\
\hline Diatoma vulgare var. vulg. Bory & $\mathrm{X}$ & $\mathrm{X}$ & & $x$ & 50 & c \\
\hline Fragilaria capucina Desm. & $\mathrm{X}$ & $\mathrm{X}$ & $\mathrm{X}$ & & 18.75 & c \\
\hline Fragilaria sp. & & $\mathrm{X}$ & & & 6.25 & $\mathbf{r}$ \\
\hline Synedra acus Kütz. & $X$ & $\mathrm{X}$ & $X$ & $x$ & 43.75 & c \\
\hline Synedra ulna (Nitz.) Ehr. & $\mathrm{X}$ & $\mathrm{X}$ & $\mathrm{X}$ & $x$ & 100 & C \\
\hline $\begin{array}{l}\text { Rhaphidinae } \\
\text { Achnanthaceae }\end{array}$ & & & & & & \\
\hline Achnanthes exigua Grunow & & & $X$ & & 6.25 & $\mathbf{r}$ \\
\hline Achnanthes lanceolata (Bréb.) Grun. & $X$ & $x$ & $\mathrm{X}$ & & 25 & c \\
\hline Achnanthes minutissima Kütz. & $\mathrm{X}$ & $\mathrm{X}$ & & & 25 & c \\
\hline $\begin{array}{l}\text { Cocconeis placentula Ehren. } \\
\text { Naviculaceae }\end{array}$ & $\mathrm{X}$ & $x$ & $\mathrm{X}$ & $x$ & 43.75 & c \\
\hline Amphora veneta Kütz. & & $\mathrm{X}$ & & & 12.5 & c \\
\hline Amphora sp. & & $\mathrm{X}$ & & & 6.25 & $\mathbf{r}$ \\
\hline Craticula cuspidata (Kütz.) Mann & $X$ & & & & 6.25 & $\mathbf{r}$ \\
\hline Cymbella amphicephala Nägeli & & $x$ & & $x$ & 12.5 & c \\
\hline Cymbella affinis Kütz. & $\mathrm{X}$ & $x$ & $\mathrm{X}$ & & 43.75 & c \\
\hline
\end{tabular}


Table 2. Continued...

\begin{tabular}{|c|c|c|c|c|c|c|}
\hline \multicolumn{7}{|c|}{ Sampling sites Relative } \\
\hline \multirow{2}{*}{$\begin{array}{c}\text { Taxones } \\
\text { Cymbella cistula (H.\& Ehren.) O. Kir. }\end{array}$} & \multirow[t]{2}{*}{$\mathbf{v}_{1}$} & \multirow{2}{*}{$\frac{V_{2}}{X}$} & \multirow[t]{2}{*}{$\mathbf{P}_{1}$} & \multirow{2}{*}{$\frac{P_{2}}{X}$} & \multicolumn{2}{|c|}{ frequency $(\%)$} \\
\hline & & & & & 18.75 & c \\
\hline Cymbella cymbiformis Agardh & & $x$ & $x$ & & 18.75 & c \\
\hline Cymbella minuta Hilse & $\mathrm{x}$ & & $x$ & & & c \\
\hline Cymbella prostrata (Berk.) Cleve & & $\mathrm{x}$ & $\mathrm{x}$ & $\mathrm{x}$ & 50 & c \\
\hline Denticula kuetzingii Grun. & & $x$ & & & 6.25 & $\mathrm{r}$ \\
\hline Diploneis smithii (Brébisson) Cleve & $\mathrm{x}$ & & $\mathrm{x}$ & & 31.25 & c \\
\hline Encyonema minutum (Hilse ex Rabh.) & $\mathrm{x}$ & & & & 6.25 & $\mathrm{r}$ \\
\hline Gomphoneis sp. & $\mathrm{x}$ & $\mathrm{x}$ & $x$ & & 25 & c \\
\hline Gomphonema acuminatum Ehr. & $\mathrm{x}$ & $\mathrm{x}$ & $x$ & $\mathrm{x}$ & 43.75 & c \\
\hline Gomphonema gracile Ehr. & & & $x$ & & 6.25 & $r$ \\
\hline Gomphonema parvulum (Kütz.) Kütz. & $\mathrm{x}$ & $\mathrm{x}$ & $x$ & $\mathrm{x}$ & 62.50 & c \\
\hline Gyrosigma accuminatum (Kütz.) Rabh. & $x$ & $x$ & $x$ & & 37.50 & c \\
\hline Navicula capitatoradiata Germ. & $\mathrm{x}$ & & $x$ & $\mathrm{x}$ & 31.25 & c \\
\hline Navicula decussis Östrup & $x$ & & $x$ & & 18.75 & c \\
\hline Navicula exigua Greg. ex Grun. & $x$ & & & & 6.25 & $\mathrm{r}$ \\
\hline Navicula mutica var. tropica Husted & & $x$ & & & 12.50 & c \\
\hline Navicula menisculus Schum. & $x$ & & $x$ & & 12.50 & c \\
\hline Navicula radiosa Kütz. & $\mathrm{x}$ & $\mathrm{x}$ & $x$ & $\mathrm{x}$ & 50 & c \\
\hline Navicula tripunctata (O.F.Müll.) Bory & $x$ & $x$ & $x$ & $x$ & 68.75 & C \\
\hline Navicula sp. & $x$ & & $x$ & & 31.25 & c \\
\hline Neidum affine (Ehr.) Cleve & & & $x$ & & 6.25 & $r$ \\
\hline Pinnularia abaujensis (Pant.) Ross & & $x$ & & & 12.5 & c \\
\hline Pinnularia divergens W. Smith & & & & $x$ & 6.25 & $\mathrm{r}$ \\
\hline Pinnularia divergentissima (Grun.) Cl. & & $x$ & & & 6.25 & $\mathrm{r}$ \\
\hline Pinnularia $\mathrm{sp}_{2}$ & & $x$ & & & 6.25 & $r$ \\
\hline $\begin{array}{l}\text { Reimeria uniseriata Sala et al. } \\
\text { Epithemiaceae }\end{array}$ & $x$ & & $x$ & & 25 & c \\
\hline Epithemia sorex Kütz. & $x$ & $x$ & $x$ & & 25 & c \\
\hline Epithemia turgida Kütz. & $x$ & & $x$ & & 31.25 & c \\
\hline $\begin{array}{l}\text { Rhopalodia gibba (Ehr.) Müller } \\
\text { Nitzschiaceae }\end{array}$ & $x$ & $x$ & $x$ & $x$ & 37.50 & c \\
\hline Nitzschia acicularis (Kütz.) W. Smith & $\mathrm{x}$ & $\mathrm{x}$ & $x$ & $\mathrm{x}$ & 43.75 & c \\
\hline Nitzschia amphibia Grun. & $x$ & $x$ & & $x$ & 18.75 & c \\
\hline Nitzschia linearis (Ag.) W. Smith & $x$ & $\mathrm{x}$ & & & 12.5 & c \\
\hline Nitzschia palea (Kütz.) W. Smith & $x$ & $x$ & $x$ & & 18.75 & c \\
\hline Surirellaceae & & & & & & \\
\hline Cymatopleura solea (Bréb.) W. Smith & & $\mathrm{x}$ & $x$ & & 18.75 & c \\
\hline Surirella angusta Kütz. & & $x$ & & & 6.25 & c \\
\hline Surirella ovalis Brèb. & $\mathrm{x}$ & $x$ & $x$ & & 25 & c \\
\hline
\end{tabular}

Table 3. Ecological attributes of algal and cyanobacterial communities calculated with average values obtained along the water cycle in the two studied sub-basins. Sampling sites: El Volcán $\left(\mathbf{V}_{1}\right)$, Cuchi Corral River $\left(\mathbf{V}_{2}\right)$, Los Molles River $\left(\mathbf{P}_{1}\right)$ and Las Chacras River.

\begin{tabular}{|c|c|c|c|c|}
\hline & $v_{1}$ & $\mathbf{v}_{2}$ & $P_{1}$ & $\mathbf{P}_{2}$ \\
\hline$\alpha$ Diversity & 48 & 58 & 50 & 39 \\
\hline Shannon and Wiener $\left(\mathrm{H}^{\prime}\right)$ & 3.947 & 4.759 & 4.133 & 2.742 \\
\hline$\beta$ Diversity (Complementarily Index) & \multicolumn{2}{|c|}{0.51} & \multicolumn{2}{|c|}{0.75} \\
\hline Jaccard's index of similarity & \multicolumn{2}{|c|}{0.49} & \multicolumn{2}{|c|}{0.28} \\
\hline
\end{tabular}

\subsubsection{El Volcán river sub-basin}

While station $\mathbf{V}_{1}$ presented a richness of 48 species, in which $39 \%$ of them were Bacillariophyceae,
58 species were found in station $\mathbf{V}_{2}$, with a $60 \%$ increase in Bacillariophyceae, at the expense of Chlorophyceae and Dinophyceae, and the presence 


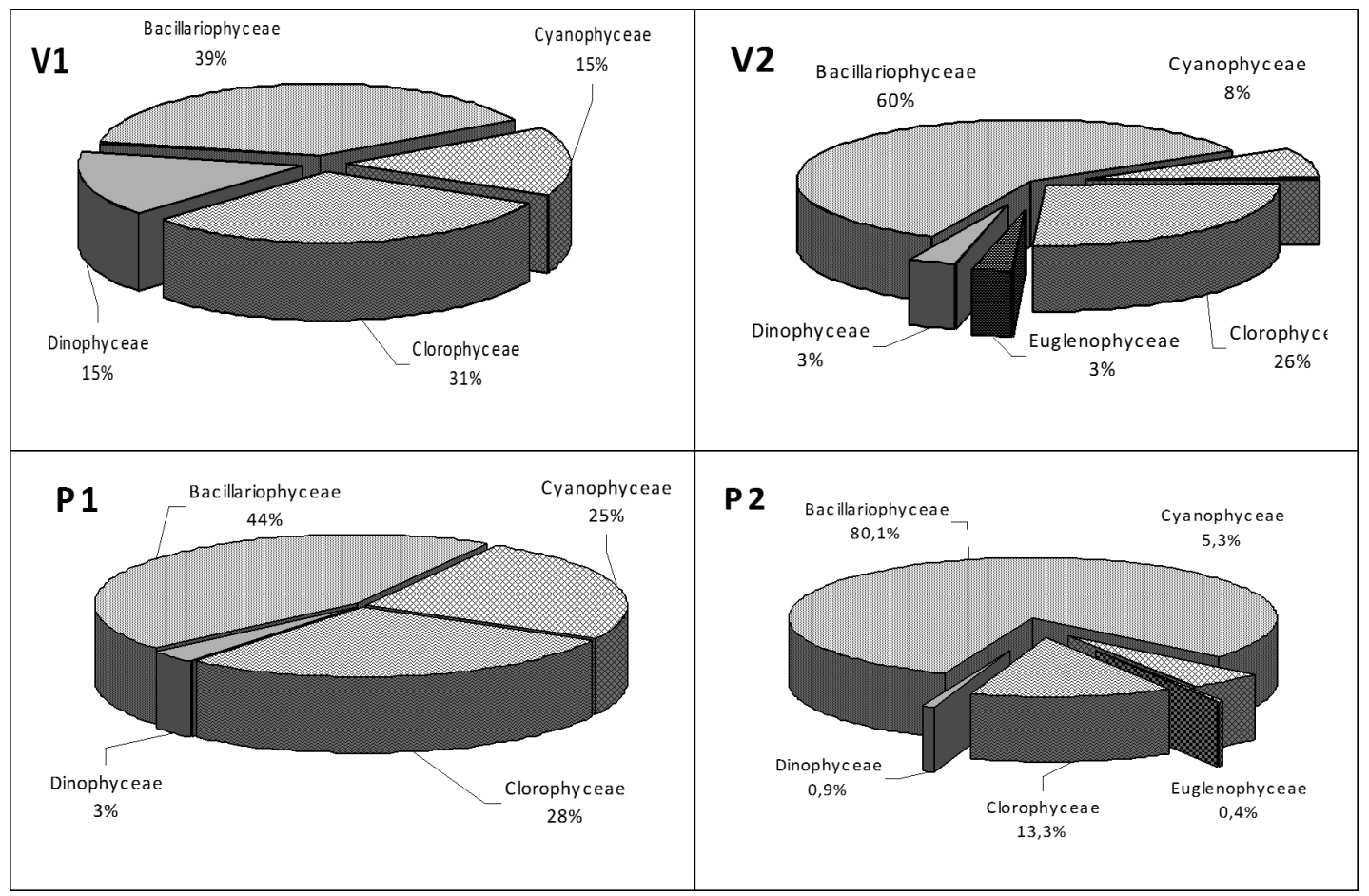

Figure 2. Relative abundance of different class of algae at the sampling sites in the Bebedero Basin (San Luis). $V_{1} E l$ Volcán River, $\mathrm{V}_{2}$ Cuchi Corral River, $\mathrm{P}_{1}$ Los Mollles River and $\mathrm{P}_{2}$ Las Chacras River.

of $3 \%$ of Eugnophyceae (Figure 2). This increase was also reflected in the specific diversity $\left(H^{\prime}\right)$ that arises from 3.95 to 4.76 bits.ind $^{-1}$.

An increase in the stream width and a decrease in the speed of the current were observed during the autumn sampling, which caused stagnation of water. A proliferation of dinoflagellate Ceratium hirundinella was also observed during this season; while Oscillatoria limosa was observed in winter.

Station $\mathbf{V}_{2}$ was placed after a gravel removal site; this produced an increase in finer sediments, and turbidity. A decrease in phytoplankton abundance was observed in the station located after the dam, especially due to the decrease of Synedra ulna, Gomphonema parvulum, Cladophora glomerata, Oscillatoria limosa, and to the disappearance of Ceratium hirundinella.

The $\beta$ diversity and the lj showed that the two stations are different in a 50-49\% respectively (Table 3), while the statistical comparison showed that they are significantly different $(\mathrm{p}=0.0008$; $\mathrm{u}=862,0)$.

\subsubsection{Potrero river sub-basin}

In spring, the presence of ashes produced as a result of large fires in the mountains was registered in station $\mathbf{P}_{1}$, mainly at stream heads. $\mathbf{P}_{1}$ presented a richness of 50 species - 44\% were Bacillariophyceace - while in the station $\mathbf{P}_{2}, 39$ species diminished $-80 \%$ of them were Bacillariophyceace with a remarkable decrease in the rest of species.

Euglenophyceae was registered in $\mathbf{P}_{2}$ at $0.4 \%$ (Figure 2), and the proliferation of Cyanophyceae - which doubles its richness in relation to the reference station - was observed. This variation also corresponds to the reduction in the value of species diversity ( $\left.\mathrm{H}^{\prime}\right)$, and reveals a less equitable community in the station located after the dam

Phytoplankton abundance was higher, and there was an increase in the station located after the dam from 290 to 639 ind. $\mathrm{mL}^{-1}$, especially due to the increase of Melosira varians, Navicula tripunctata, and Synedra ulna, and to the appearance of Coelastrum microporum.

The diversity $\beta$ and the $\mathrm{lj}$ showed that the two stations are different in a $75-28 \%$ respectively (Table 3), while the statistical comparison did not show important difference $(\mathrm{p}=0.766$; $\mathrm{u}=938.5)$.

\section{Discussion}

The sites studied present algal communities with high diversity and low abundance. This situation is common to other streams in the 
area (Martínez de Fabricius et al., 2007). All the species reflected low values of frequency, with the exception of some diatoms, such as Synedra ulna and Gomphonema parvulum. These two species were found in four sampling stations, and they were pointed out as species adapted to a wide range of conditions, and highly tolerant to waters with high organic matter concentration (Salomoni et al., 2006). A better phytoplankton structure was observed in the head station that was only interrupted in station $\mathbf{P}_{\mathbf{1}}$, where a great fire affected this area.

Euglenophytas form a group related to contamination with organic matter, and a qualitatively well represented group in lentic environments. They were present in the post-dam sites, and they are subjected to the anthropogenic influence, revealing that they are good indicators of water quality (Reynolds, 1997; Reynolds et al., 2002, Mirande et al., 2005).

The lowest density values were registered during summer at the Cuchi Corral river. This fact is related to the presence of sediments, which produce a turbidity that reduces the penetration of light - a well known factor that limits the development of phytoplankton -, thus generating an unstable environmental situation and preventing a good development. This effect has already been acknowledged in the bibliography (CARP, 1989; Bonilla and Conde, 2000). The presence of Artrospira sp - only local cyanophyteswould be related to the maximum tolerance to highly mineralized environments and with high conductivity. All those conditions were found in this site. This parameter is frequently measured and used as geochemical index for the control of natural waters (Espinosa et al., 1999).

Variation and reduction of phytoplankton in $\mathbf{P}_{\mathbf{1}}$ could be influenced by the fires that happened in this zone in spring. According to Holopainen and Huttunen (1992), changes in water quality, such as the increase in the washing of nutrients in disturbed zones, causes changes in the algal flora. These authors found that there is a diversity of more than 63 algae taxa, and that dominant groups change. In our case, there was a remarkable decrease in the composition and abundance of phytoplankton. If these areas are not properly protected, there could be an increase of nutrients (eutrophication) that impact on the body water receptors developing different forms of aquatic vegetation (phytoplankton, periphyton, etc.) that are not characteristic of the environment (Temporetti, 2006). Bacillariophyceae, in particular species of the Pennales Order, were both qualitatively and quantitatively predominant. Central Order species, on the other hand, had little representation, except for the central diatom Melosira varians which predominated in $\mathbf{P}_{2}$, characterized as a primary colonization agent in environments with low flow conditions, accumulation of organic matter and nutrients in the water (Martínez, 2003; Seeligman et al., 2001). These results differ from the observations by Daruich (2007), but agree with those by Molloy (1992), who asserts that central diatoms are more frequent downstream. According to Álvarez-Cobelas and Rojo (1994), the increase in central populations is related to high eutrophic systems, which might be happening in $\mathbf{P}_{2}$.

The success of diatoms is due to high photosynthetic effectiveness, high amount of chlorophyll and low threshold of light saturation, features that give them adaptive advantages over other components of phytoplankton (Reynolds, 1988), in addition to their capacity of living in highly unstable environments and of quickly responding to environmental changes in fluvial unstable systems (Rojo et al., 1994). Transparency and stability of water column in $\mathbf{V}_{1}$ and $\mathbf{P}_{1}$, as well as the decrease of flow determined the diminution of diatoms as a result of the increase in their sinking rate and their replacement by green algae (Margalef, 1993). It is interesting to note the proliferation of Ceratium hirundinella in $\mathbf{V}_{1}$ during autumn. This dinoflagellate has been found in Argentine dams, and it extends from south to north with no particular explanation, a situation that could be related to important environmental alterations (Boltovskoy et al., 2003). The most affected station was $\mathbf{P}_{2}$, showing high abundance with less diversity and equitability. The most tolerant species to organic matter, such as Melosira varians, Navicula tripunctata and Oscillatoria limosa are predominant in autumn and winter, whereas Coelastrum microporum predominates in summer. According to Dolbeth et al. (2003) and Western (2001), all this suggests an evolution in the eutrophic process from an initial to an intermediate level, or what is more, from an intermediate to an advanced level of this process. Consequently, the chemical evaluation of water quality in the Potrero river showed deterioration signs downstream from the village, with an increase in the organic matter (Almeida et al., 2007). Although Coelastrum microporum was found in all types of lakes in Argentina (Mirande et al., 2009), particularly in cool lakes with important anthropogenic disturbances 
(Vila and Pardo, 2003). The occurrence of this species during summer in the $\mathbf{P}_{2}$ reveal that it originates in dams with eutrophication signs.

\section{Conclusion}

The phytoplankton community was mainly composed of diatoms and chlorophytes on a smaller scale, with some downstream variations as a response to aquatic environment conditions. A remarkable change in the number and specific composition of phytoplankton was produced in the disturbed sites, and species more tolerant to cyanophytes appeared in their place. Euglenophytes - which have an affinity with organic matter coming from the dam - appeared in the post-dam station, and the diatoms such as Melosira varians and Navicula tripunctata were also present. All the species reflected low values of frequency, with the exception of some diatoms, such as Synedra ulna and Gomphonema parvulum, which are considered as species tolerant to a wide range of ecological conditions. Therefore, the structure and composition of the algal and Cyanobacterial communities allowed us to identify sections more sensitive to human-induced alterations.

\section{Acknowledgments}

The present study was financed by the Secretaria de Ciencia y Tecnica from the Universidad Nacional de San Luis. Argentina.

\section{References}

ALMEIDA, CA., QUINTAR, S., GONZÁLEZ, P. and MALLEA, MA. 2007. Influence of urbanization and tourist activities on the water quality of the Potrero de los Funes River (San Luis - Argentina). Environmental Monitoring and Assessment, vol. 133, no. 1-3, p. 45965. http://dx.doi.org/10.1007/s10661-006-9600-3

ÁLVAREZ-COBELAS, M. and ROJO, C. 1994. Factors influencing the share of planktic diatotns in lakes. Archiv für Hydrobiologie - Supplement, vol. 74, p. 73-104.

ALVEAL, K., FERRARIO, MS., OLIVEIRA EC. and SAR, F., eds. 1995. Manual de métodos ficológicos. Concepción: Universidad de Concepción. 863 p.

ANDERSON, DL., DEL AGUILA, JA. and BENARDON, AE. 1970. Las formaciones vegetales en la provincia de San Luis. Revista de Investigaciones Agropecuarias, vol. 7, no. 3, p. 153-182.

BONILLA, S. and CONDE, D. 2000. El fitoplancton como descriptor sensible de cambios ambientales en las lagunas costeras de la Reserva Bañados del Este. In Seminario-Taller sobre monitoreo ambiental, 1998. Rocha: Probides. vol. 31, p. 63-74.
BOLTOVSKOY, A., ECHENIQUE, R. and GUERRERO, JM. 2003. Colonización de limnotopos de Argentina por Ceratium hirundinella (Dinophyceae). Boletín de la Sociedad Argentina de Botánica, vol. 38, p. 148. Supplement.

BOURRELY, P. 1981. Les Algues d'eau douce. Les algues jaunes et brune. II. París: Ed. Boubée.

BOURRELY, P. 1985. Les Algues d'eau douce. Les algues bleues et rouges. III. París: Ed. Boubée.

BOURRELY, P. 1990. Les Algues d'eau douce. Les algues vertes. I. París: Ed. Boubée.

CABRERA, A. 1976. Regiones fitogeográficas argentinas. In LORENZO, R. Enciclopedia Argentina de Agricultura y Jardinería. Buenos Aires: Ed. Acme S.A.I.C. 81 p.

Comisión Administradora del Río de la Plata - CARP. 1989. Estudio para la evaluación de la contaminación en el Río de la Plata. Informe de Avance. 422 p.

CECI, JH. and CRUZ CORONADO, MD. 1981. Recursos Hídricos Subterráneos. In Geología de la Provincia de San Luis, VIII Congreso Geológico Argentino Rel, 1981. p. 301-322.

COLWELL, RK. and CODDINGTON, JA. 1994. Estimating terrestrial biodiversity through extrapolation. Philosophical Transactions of the Royal Society of London, Series B, vol. 345, p. 101-118. http://dx.doi.org/10.1098/rstb.1994.0091

COX, E.J. 1991. What is the Basis for using Diatoms as Monitors of River Quality?. In WHITTON, BA., ROTT, E. and FRIEDRICH, G., eds. Use of Algae for Monitoring Rivers. Innsbruck: University. p. 33-41.

DARUICH, J. and MARTÍNEZ DE FABRICIUS, AL. 2000. Estudios preliminares de las comunidades algales de la cuenca del río Nogolí (Dep. Belgrano, Prov. San Luis, Argentina). In Anais da XVIII Reunión Argentina de Biologia CUYO, 2000. p. 58.

DARUICH, J. and MARTÍNEZ DE FABRICIUS, AL. 2001. Fitoplancton del río Nogolí (Prov San Luis). Boletín de la Sociedad Argentina de Botánica, vol. 36, p. 58. Supplement.

DARUICH, J., NIEVAS, CM. and MARTÍNEZ DE FABRICIUS AL. 2003. Variación en la composición de Diatomeas de la Cuenca del río Nogolí (San Luis, Argentina). Boletín de la Sociedad Argentina de Botánica, vol. 38, p. 150. Supplement.

DARUICH, J., MARTÍNEZ DE FABRICIUS, A. and ULACCO, JH. 2005. Epilithic algae from Nogoli River (San Luis, Province). BIOCELL, vol. 29, no. 3, p. 360.

DARUICH, J. and MARTÍNEZ DE FABRICIUS, AL. 2006. Diatomeas de la Cuenca del río Nogolí. Su distribución en un ciclo anual (San Luis, Argentina). In Anais do II Congreso Peruano de Ficología, 2006. 
DARUICH, J. 2007. Composición y Distribución de las Bacillariophyceae de la cuenca del río Nogolí, San Luis, Argentina. San Luis: Universidad Nacional de San Luis. [Doctoral thesis in Biological Sciences].

DESIKACHARY, TV. 1959. Cyanophyta. New Delhi: Indian Council of Agricultural Research, Editorial BOARD. 686 p.

DOLBETH, M., PARDAL, MA., LILLEBLO, AI., AZEITEIRO, U. and MARQUES, JC. 2003. Short-and long-term effects of eutrophication on the secondary production of an intertidal macrobenthic community. Marine Biology, vol. 10, no. 1007, p. 1133-1135.

ESPINOSA, MC., GONZÁLEZ, P., FAGUNDO, JR., SUÁREZ, M., BENÍTEZ, G., RAMÍREZ, J. and MAYARÍ, R. 1999. Empleo de índices geoquímicos en el establecimiento del monitoreo de cultivos de microalgas. In Cuba. Ministerio de Ciencias Tecnología y Medio Ambiente. Contribución y Educación a la Protección Ambiental. La Habana: ISCTN. p. 163-167.

GERMAIN, H. 1981. Flore des diatomées. Paris: Societé Nouvelle des éditions Boubée. 444 p.

HOLOPAINEN, AL. and HUTTUNEN, P. 1992. Effects of Forest Clear-catting and Soil Disturbans on the Biology of Small Forest Group. Hydrobiologia, vol. 243-244, p. 457-464. http://dx.doi.org/10.1007/ BF00007063

KRAMMER, K. and LANGE-BERTALOT, H. 1986. Bacillariophyceae 2. Naviculaceae. 2/1. G. F. Verlag, Jena. 875 p.

KRAMMER, K. and LANGE-BERTALOT, H. 1988. Bacillariophyceae 2. Bacillariaceae. Ephitemiaceae. Surirellaceae 2/2. G. Fisher, Stuttgart. New York. 596 p.

KRAMMER, K. and LANGE-BERTALOT, H. 1991 a. Bacillariophyceae 3. Centrales. Fragilariaceae. Eunotiaceae 2/3. G. Fisher, Stuttgart. New York. 576 p.

KRAMMER, K. and LANGE-BERTALOT, H. 1991 b. Bacillariophyceae 4. Achnantaceae 2/4. G. Fisher, Stuttgart. New York. 437 p

MAIDANA, N. and HERBEST, N. 1994. Diatomeas del Chaco (Argentina): II. Boletín de la Sociedad Argentina de Botánica, vol. 30, no. 1-2, p. 25-42.

MARGALEF, R. 1993. Teoría de los sistemas ecológicos. Publications Universitat de Barcelona. 1010 p.

MARTÍNEZ, LF. 2003. Efectos del caudal sobre la colonización de algas en un río de alta montaña tropical (Boyacá, Colombia). Caldasia, vol. 25, n. 2, p. 337-354.

MARTÍNEZ DE FABRICIUS, AL. 2000. Bacillariophyceae del río Cuarto, provincia de Córdoba (Argentina), Fam. Thalassiosiraceae, Melosiraceae y Diatomaceae. Boletín Sociedad Argentina de Botánica, vol. 35, no. 1-2, p. 33-48.
MARTÍNEZ DE FABRICIUS, AL., MAIDANA, N., GÓMEZ, N. and SABATER, S. 2003. Distribution patterns of benthic diatoms in a Pampean river exposed to seasonal flods: the Cuarto river (Argentina). Biodiversity and Conservation, vol. 12, p. 2443-2454. http://dx.doi.org/10.1023/A:1025857715437

MARTÍNEZ DE FABRICIUS, AL., LUQUE, ME., LOMBARDO, D. and BRUNO, E. 2007. Potamoplancton en la cuenca media del río Cuarto (Córdoba, Argentina. Limnetica, vol. 26, no. 1, p. 25-38.

MIRANDE, V. and TRACANNA, BC. 2005. Fitoplancton de un río del noroeste argentino contaminado por efluentes azucareros y cloacales. Boletin de la Sociedad Argentina de Botánica, vol. 40, no. 3-4, p. 169-182.

MIRANDE, V., HALEBLIAN, SE., BARRETO, GA. and TRACANNA, BC. 2009. Biodiversidad del Parque Nacional Pre-Delta (Entre Ríos, Argentina). I) Riqueza del fitoplancton. Lilloa, vol. 46, no. 1-2, p. 88-136.

MOLLOY, JM. 1992. Diatom communities along stream longitudinal gradients. Freshwater Biology, vol. 28, p. 59-69. http://dx.doi. org/10.1111/j.1365-2427.1992.tb00562.x

NIEVAS, CM., DARUICH, J. and MARTÍNEZ DE FABRICIUS, AL. 2003. Estudios ficológicos preliminares del Valle del Conlara (Dep. Chacabuco, Prov. de San Luis, Argentina). Boletín de la Sociedad Argentina de Botánica, vol. 38, p. 158. Supplement.

MORENO, CE. 2001. Métodos para medir la biodiversidad. SEA. Pachuca: Editado por Cooperación Iberoamericana (CYTED), UNESCO (ORCYT) y SEA. vol. 1, 83 p.

PAREDES, C., IANNACONE, J. and ALVARIÑO, L. 2007. Biodiversidad de invertebrados de los humedales de Puerto Viejo, Lima, Perú. Neotropical Helminthology, vol. 1, no. 2, p. 21-30.

PATRICK, R. and REIMER, CW. 1966. The diatoms of the United States (exclusive of Alaska and Hawaii). Monograph Academy of Natural Sciences of Philadelphia, vol. 13, no. 1, p. 1-688.

PATRICK, R. and REIMER, CW. 1975. The Diatoms of the United States exclusive of Alaska and Hawai. Academy of Natural Sciences of Philadelphia Monographs, vol. 13, no. 2, p. 1-213.

PEREIRA, I., REYES, G. and KRAMM, V. 2000. Cyanophyceae, Euglenophyceae, Chlorophyceae, Zygnematophyceae y Charophyceae en arrozales de Chile. Gayana Botánica, vol. 57, p. 29-53. http:// dx.doi.org/10.4067/S0717-66432000000100003

PRESCOTT, GW. 1962. Algae of the Western Great lake area. N. M C. Brow. Co. Ine. 977 p.

RAMÍREZ GARCÍA, P., MARTÍNEZ ROMERO, E., MARTÍNEZ SALGADO, MD. and ESLAVA CAMPOS, CA. 2007. Cianobacterias, 
microorganismos del fitoplancton, y su relación con la salud humana. Available from: <http://www2.ine. gob.mx/publicaciones/libros/440/cap4.html>.

REYNOLDS, CS. 1988. Functional morphology and adaptative strategies of freshwater phytoplankton. In SANDGREN, C., ed. Growth and reproductive strategies of freshwater phytoplankton. London: Cambridge University Press. 210 p.

REYNOLDS, CS. 1997. Vegetation processes in the pelagic: a model for ecosystem theory. Oldendorf: Ecology Institute. $371 \mathrm{p}$.

REYNOLDS, CS., HUSZAR, V., KRUK, C., FLORES, LN. and MELO, S. 2002. Toward a functional classification of the freshwater phytoplankton. Journal of Plankton Research. vol. 24, no. 5, p. 417 428. http://dx.doi.org/10.1093/plankt/24.5.417

ROJO, C., ÁlVAREZ COBELAS, M. and ARAUZO M. 1994. An elementary, structural analysis of river phytoplankton. Hydrobiologia, vol. 289, p. 43-55. http://dx.doi.org/10.1007/BF00007407

ROUND, FE. and BUKHTIYAROVA, L. 1996. Four new genera based on Achnanthes (Achnanthidium) together with a re-definition of Achnanthidium. Diatom Research, vol. 11, no. 2, p. 345-361. http:// dx.doi.org/10.1080/0269249X.1996.9705389

SALOMONI, SE., ROCHA, O., CALLEGARO, VL. and LOBO, EA. 2006. Epilithic diatoms as indicators of water quality in the Gravatí river, Río Grande do Soul, Brazil. Hydrobiologia, vol. 559, p. 233-246. http://dx.doi.org/10.1007/s10750-005-9012-3

SEELIGMAN, C., TRACANNA, BC., MARTINEZ DE MARCO, S. and ISASMENDI, S. 2001. Algas fitoplanctónicas en la evaluación de la calidad del agua de sistemas lóticos en el noroeste argentino. Limnetica, vol. 20, no. 1, p. 123-133.

SILVA, HJ., LUCO JM., GONZÁLEZ, DM. and BAUDINO, OM. 1995. Detección de compuestos biogénicos-volátiles en un lago eutrófico de San Luis- Argentina. Acta Toxicológica Argentina, vol. 3, no. 2, p. 38-42.
SIMMONSEN, R. 1979. The Diatom Sistem, Ideas on phylogenya. Bacillaria, vol. 2, p. 9-71.

STOERMER, EF., KREIS, RG. and ANDERSEN, NA. 1999. Checklist of Diatom from the Laurentian Great Lakes: II. Journal of Great Lakes Research, vol. 25, no. 3, p. 545-566.

TEMPORETTI, PF. 2006. Efecto a largo plazo de los incendios forestales en la calidad del agua de dos arroyos en la sub-región Andino-Patagónica, Argentina. Ecología Austral, vol. 16, p. 157-166.

TOGNELli, G., STRASSER, E., CORTINEZ, V., MORLA, P. and FERNÁNDEZ, L. 1997. Eutrofización del dique Cruz de Piedra. Departamento Capital, San Luis Argentina. In Anais do $1^{\circ}$ Congreso Latinoamericano de Sedimentología. Memorias, 1997. vol. 1, p. 299-307.

UTERMÖHL, H. 1958. Zur Vervollkommnung der quantitativen Phytoplankton-Methodik. Mitteilungen der Internationale Vereinigung für Theoretische und Angewandte Limnologie, vol. 9, p. 1-38.

VERTERAMO, A., NIEVAS, CM. and MARTÍNEZ DE FABRICIUS, AL. 2001. Estudios Ficológicos en dos ríos de la Provincia de San Luis. Boletín de la Sociedad Argentina de Botánica, vol. 36, p. 64. Supplement.

VILA, I. and PARDO, R. 2003. Respuesta de la estructura fitoplanctónica a las perturbaciones antrópicas de un lago templado. Limnetica, vol. 22, no. 1-2, p. 93-102.

VILLAFAÑE, VE. and REID, FMH. 1995. Métodos de microscopía para la cuantificación del fitoplancton. In ALVEAL, K., FERRARIO, ME., OLIVEIRA, EC. and SAR, E., eds. Manual de Métodos Ficológicos. Concepción: Edit. Universitaria. p. 169-185.

WETZEL, R. 1981. Limnología. Barcelona: Ed. Omega, S.A. 679 p.

WESTERN, D. 2001. Human-modified ecosystems and future evolution. PNAS - Proceedings of the National Academy of the United States of America, vol. 98, p. 5458-5465. 